\title{
Chromatic Dispersion Compensation in Optical Fiber Communication System and its Simulation
}

\author{
R. Udayakumar ${ }^{*}$, V. Khanaa ${ }^{2}$ and T. Saravanan ${ }^{3}$ \\ 'Associate Professor, Department of Information Technology, \\ Bharath University Chennai-600 073; ruskumar2007@hotmail.com \\ 2Dean-Information Technology, Bharath University, Chennai-600 073; \\ drvkannan62@yahoo.com \\ ${ }^{3}$ Professor \& Head, Department of ETC, Bharath University, Chennai-600073, India; \\ saravanan.etc@bharathuniv.ac.in
}

\begin{abstract}
Chromatic dispersion is a critical factor that limits the quality of transmitted optical signal in high speed fiber optic communication systems. Chromatic dispersion occurs due to the propagation delay spread of different spectral components of the transmitted signal. This leads to the pulse broadening of transmitted signal. In order to operate the fiber optic communication system with a sufficiently low bit error rate and to minimize the performance degradation caused by pulse distortion and broadening, dispersion compensation is needed. The purpose of this project is to compensate the chromatic dispersion using fiber Bragg grating and optical phase conjugation techniques. Simulation results will be obtained using OPTSIM software version 5.3. Effect of chromatic dispersion in an optical fiber link was analyzed. Simulation using Fiber Bragg grating by varying its grating period and linear chirp coefficient was obtained.
\end{abstract}

Keywords: (DCF)Dispersion Compensating Fiber,(OPC)Optical Phase Conjugator,(FBG)Fiber Bragg Grating.

\section{Introduction}

Fiber optic communication is a method of transmitting information from one place to another by sending light through an optical fiber. The light forms an electromagnetic carrier wave that is modulated to carry information [1]. The process of communicating using fiber optics involves the following basic steps: Creating the optical signal using a transmitter, relaying the signal along the fiber, ensuring that the signal does not become too distorted or weak, and receiving the optical signal and converting it into an electrical signal.

Dispersion characterizes optical fiber in terms of maximum transmission speed. When different wavelengths of light pulses are launched into an optical fiber, these pulses will travelled at different speeds due to the variation of refractive index with wavelength. These light waves tend to get spread out in time after traveling some distance in the optical fiber and this is continued throughout the length of the fiber. This phenomenon of broadening of pulse width is called dispersion. The pulse width gradually increases and peak power of impulse is reduced. Dispersion of the transmitted optical signal causes distortion for both digital and analog transmission along optical fibers. Each pulse broadens and overlap with its neighbors eventually becoming indistinguishable at the receiver input. This effect is known as inter symbol interference.

Dispersion limits information capacity at high transmission speeds. Dispersion reduces the effective bandwidth and at the same time it escalates the error rate due to an increasing inter symbol interference. In order to remove the spreading of optical pulses, the dispersion compensation is the most important feature required in optical fiber communication system.

* Corresponding author:

R. Udayakumar (ruskumar2007@hotmail.com) 


\section{System Modeling}

Chromatic dispersion occurs due to the propagation delay differences between the different spectral components of the transmitted signal. This causes broadening of each transmitted mode. The delay differences may be caused by the dispersive properties of waveguide material (Material dispersion) and also guidance effects within the fiber structure (Waveguide dispersion).

Chromatic dispersion are of two types - Material Dispersion and Waveguide Dispersion.

Pulse broadening due to material dispersion results from the different group velocities of the various spectral components launched into the fiber from the optical source. It occurs when the phase velocity of a plane wave propagating in the dielectric medium varies non linearily with wavelength and a material is said to exhibit material dispersion when the second differential of the refractive index with respect to wavelength is not zero [2]. The pulse spread due to material dispersion may be obtained by considering the group delay $\tau_{g}$ in the optical fiber which is the reciprocal of the group velocity $\vartheta_{g}$.

Waveguide dispersion depends upon the geometry of the fiber which confines the light into the core of an optical fiber. Due to the smaller diameter of the SMF it confines $80 \%$ of the light into the core of the fiber while the remaining $20 \%$ travels along the cladding with a speed faster than in the core and is lost but in multimode fiber due to the larger diameter almost all the power is confined to the core so waveguide dispersion mainly occurs in Single mode fiber. It is usually neglected during the operation of the optical fiber communication.

Group velocity dispersion (GVD) causes the light pulses to spread in fibers, degrading signals over long distances. It causes a short pulse of light to spread in time as a result of different frequency components of pulse travelling at different velocities. Group velocity dispersion can be reduced by producing solitons. Soliton refers to special kind of waves that can propagate undistorted over long distances and remain unaffected after collisions with each other.

Different methods are used to compensate chromatic dispersion. Dispersion compensating fiber [3] (DCF) is one method used to compensate dispersion. It is a simple and reliable method. But DCF have high attenuation and high insertion loss [4]. Due to these disadvantages DCF is replaced by Fiber Bragg Grating (FBG) and Optical Phase Conjugator (OPC).

\subsection{Proposed Method}

Figure 1 shows the block diagram for compensating the dispersion using fiber Bragg grating [5] and optical phase conjugator. The proposed method analyzed the effect of dispersion in the fiber optic link and then compensates the dispersion using fiber Bragg grating and optical phase conjugator. Finally comparing the eye diagrams and bit error rate of the two methods.

\subsection{Simulation layout}

Figure 2 shows the simulation layout of Fiber Bragg Grating. The transmitter section consists of a PRBS signal generator, electrical signal generator, laser source and Mach- zehnder modulator. The PRBS signal generator generates a pseudo random binary sequence. The electrical signal converts the logical input signal, a binary sequence of zeros and ones from PRBS generator into an electrical signal. The laser source generates laser beam. This model produces the optical signal output of one or more CW lasers. It is most commonly used in conjunction with the external modulator model to encode a binary signal upon the CW source. Then it is fed into the modulator [6]. Then it is transmitted

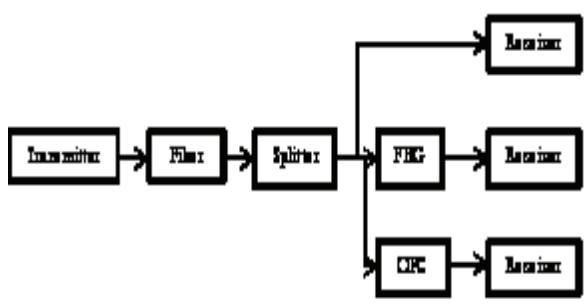

Figure 1. Block diagram using FBG and OPC.

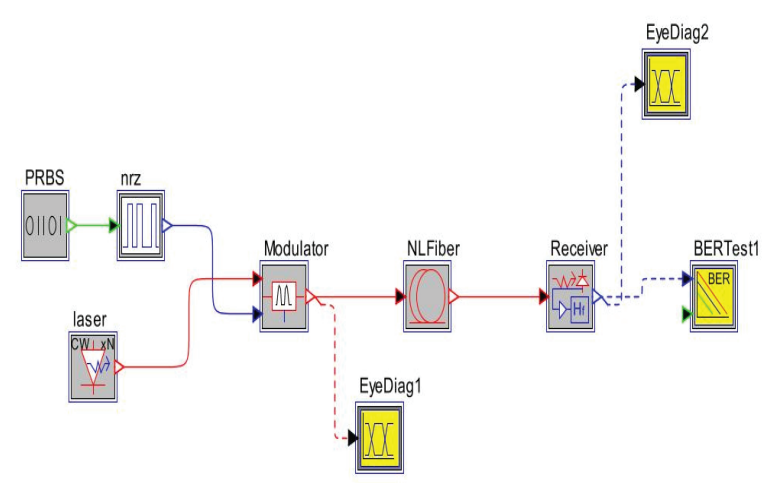

Figure 2. Simulation layout to determine the dispersion in optical fiber. 


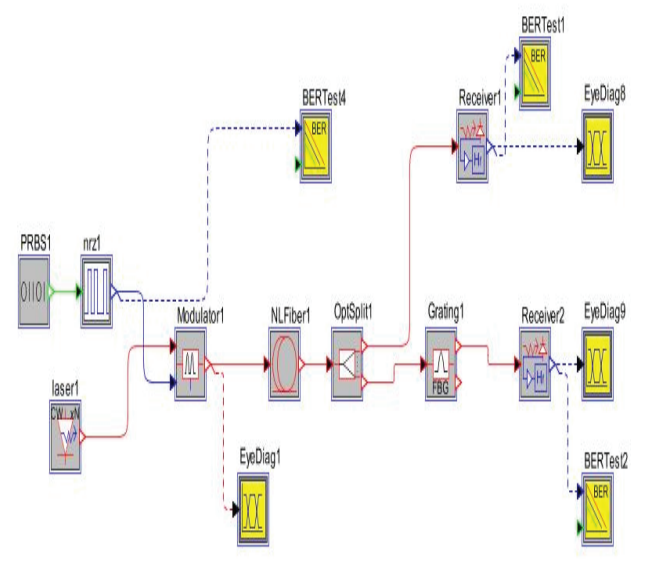

Figure 3. Simulation Layout using Fiber Bragg Grating.

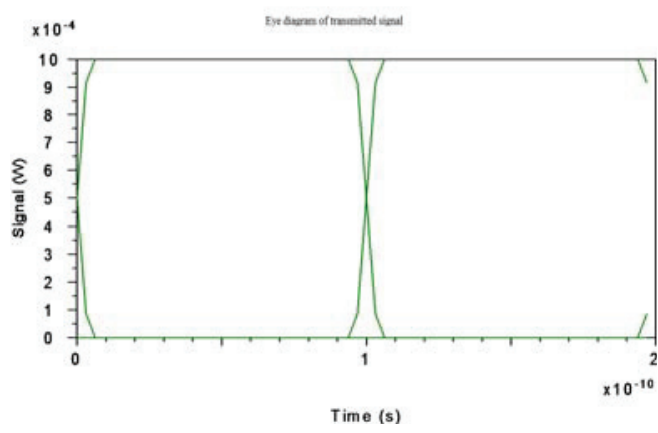

Figure 4. Eye diagram of Input signal.

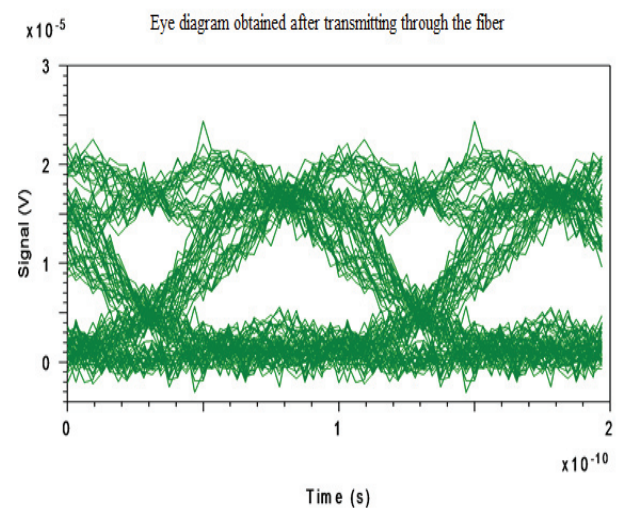

Figure 5. Eye diagram of signal after transmitting through the fiber.

through the nonlinear fiber and is splitted by an optical splitter. Finally the two outputs are obtained at the receiver with and without using fiber Bragg grating. Results are

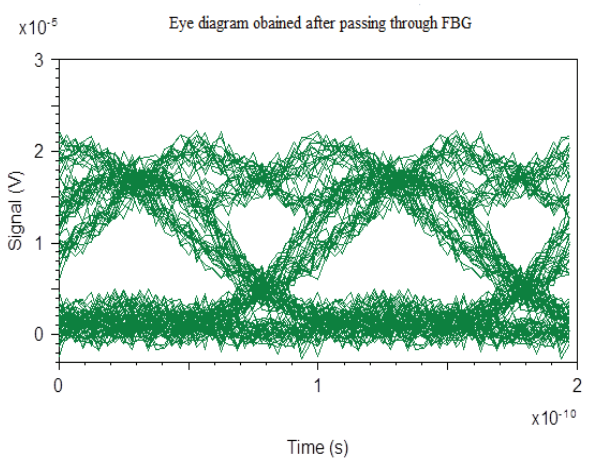

Figure 6. Eye diagram obtained after transmitting through FBG.

Table 1. Bit error rate of the signal before and after transmitting through the fiber

\begin{tabular}{|c|c|c|}
\hline Parameter & Without grating & With grating \\
\hline Bit error rate & $1.0288 \mathrm{e}-10$ & $1.4545 \mathrm{e}-12$ \\
\hline
\end{tabular}

obtained with fiber Bragg grating by varying its grating period and linear chirp coefficient using eye diagrams.

\subsection{Simulation Results}

From the Figure 4 we concluded that the eye diagram of input signal is $12 \mathrm{~V}$. The Figure 5 shows signal of transmitting waves after through the fiber. This shows the error signal is more compare to before transmitting through the fiber. The Figure 6 shows Eye diagram obtained after transmitting through FBG. The Figure 7 shows Eye diagrams obtained at different grating periods with different stages. The figure 8 shows Eye diagrams obtained at different linear chirp coefficients.

From the Table 1, it is concluded that as the bit error rate decreases the dispersion also decreases.

Table 2 is concludes that as grating period decreases the dispersion decreases.

From the table 3 it is concluded that as linear chirp coefficient increases the dispersion decreases.

\section{Conclusion}

Different dispersion techniques in optical fiber communication system are studied. Effect of dispersion in fiber optic link was analyzed using eye diagrams. We also studied the different techniques used to compensate the chromatic 

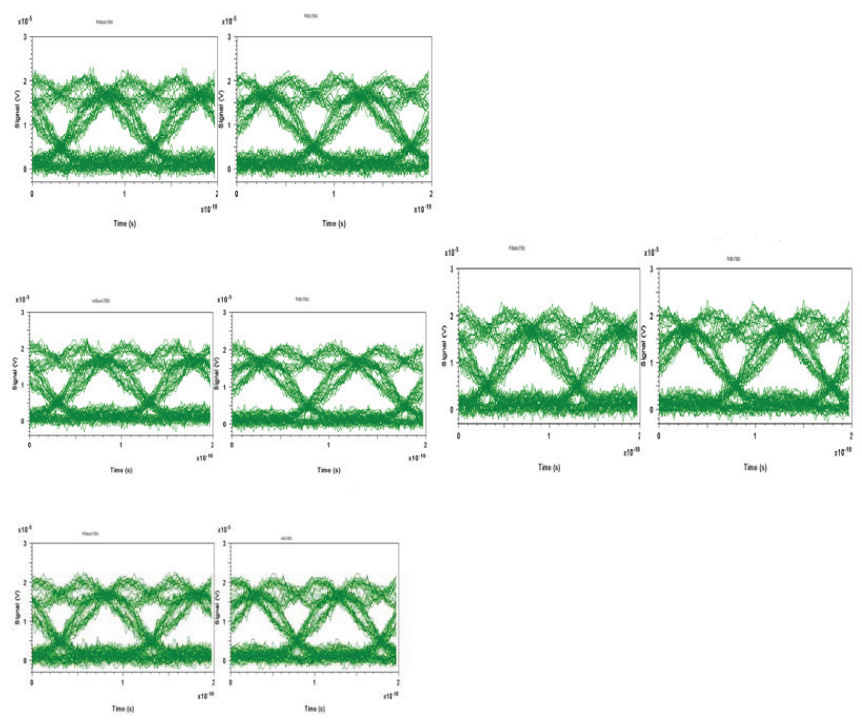

Figure 7. Eye diagrams obtained at different grating periods.

Table 2. The bit error rate with and without using FBG by varying its grating period

\begin{tabular}{|c|c|c|}
\hline Grating period & BER without FBG & BER with FBG \\
\hline $4.7562 \mathrm{e}-9$ & $1.0288 \mathrm{e}-10$ & $1.4213 \mathrm{e}-12$ \\
\hline $1.9845 \mathrm{e}-12$ & $1.0288 \mathrm{e}-10$ & $1.4214 \mathrm{e}-12$ \\
\hline $5.3421 \mathrm{e}-15$ & $1.0288 \mathrm{e}-10$ & $1.4522 \mathrm{e}-12$ \\
\hline $3.2548 \mathrm{e}-14$ & $1.0288 \mathrm{e}-10$ & $1.4316 \mathrm{e}-12$ \\
\hline
\end{tabular}

Table 3. The bit error rate with without using grating at different linear chirp coefficient

\begin{tabular}{|c|c|c|}
\hline Linear chirp coeff. & Without grating & With grating \\
\hline 0.1 & $1.0288 \mathrm{e}-10$ & $1.4530 \mathrm{e}-12$ \\
\hline 1 & $1.0288 \mathrm{e}-10$ & $1.4672 \mathrm{e}-12$ \\
\hline 1.5 & $1.0288 \mathrm{e}-10$ & $1.6808 \mathrm{e}-12$ \\
\hline 3 & $1.0288 \mathrm{e}-10$ & $8.7056 \mathrm{e}-12$ \\
\hline
\end{tabular}

dispersion. Simulation model for dispersion compensation using fiber Bragg grating is developed. Bit error rate of signal before and after transmitting through the fiber Bragg grating was compared by varying its grating period and linear chirp coefficient.

\section{References}

1. Thirumaran S, DeviS KN etal. (2012). International Conference on Devices, Circuits and Systems (ICDCS), 34-37.
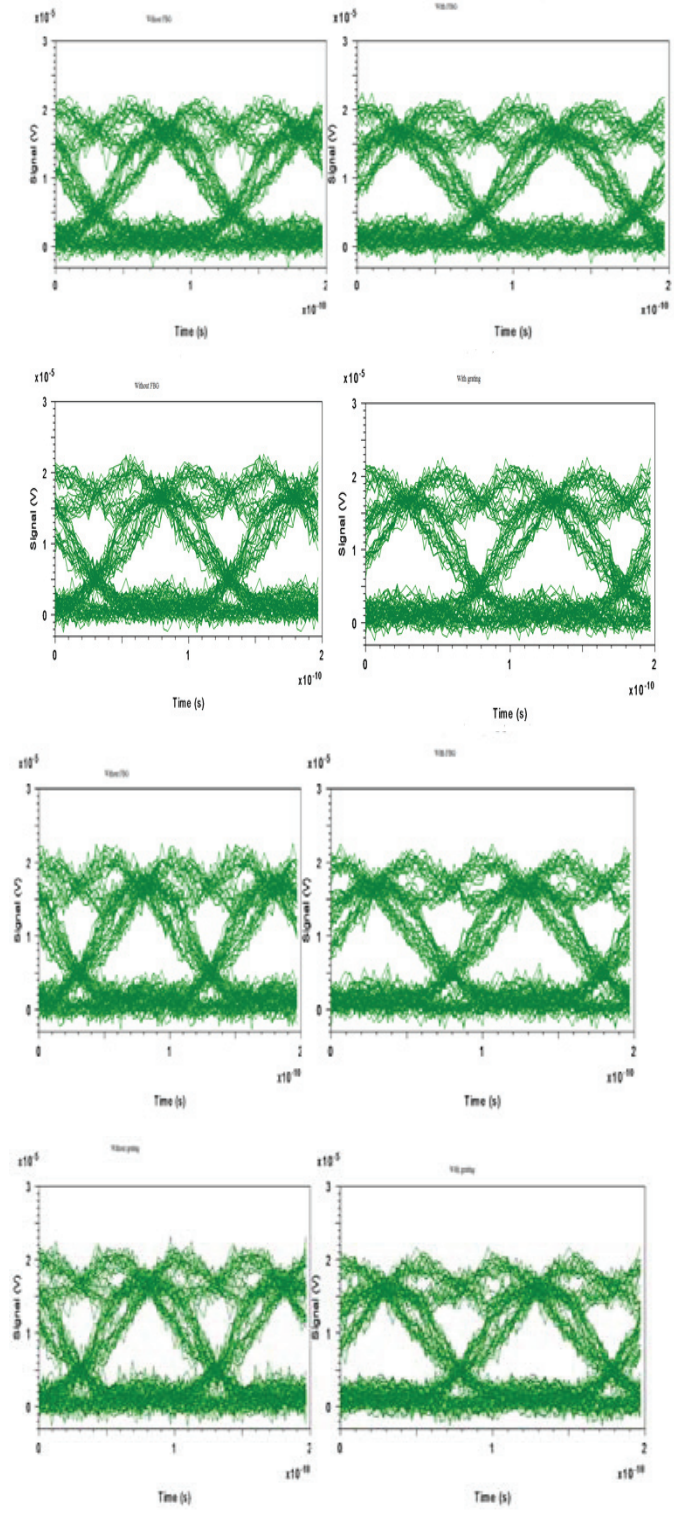

Figure 8. Eye diagrams obtained at different linear chirp coefficients.

2. Stepanek L (2012). Chromatic dispersion in optical communications, Journal of Electrical and Electronic Engineering, vol VII, No. 2, 142-151.

3. Dabhade S S, and Bhosale S (2012). Fiber bragg grating and optical phase conjugator as dispersion compensator, International Journal of Advanced Electrical and Electronics Engineering, vol 1(1), 15-19.

4. Bhowmik K, Ahamed M et al. (2012). Reduction of dispersion in optical fiber communication by fiber bragg grating and optical phase conjugation techniques, International Journal of Mobile Network Communications \& Telematics (IJMNCT), vol 2, No.3, 49-58. 
5. Arora O, Punia S et al. (2011). Symmetrical dispersion compensation for high speed optical links, International Journal of Computer Science Issues, vol 8(6), No.1, 371-376.
6. Spolitis S, Bobrovs V et al. (2011). Realization of combined chromatic dispersion compensation methods in high speed WDM Optical transmission Systems, Department of Telecommunications Engineering, vol 10, 33-38. 